\title{
Effect of Rate and Quantum Yield of 1-alkyl-2-(arylazo) Imidazoles on the Surface of Silver NPs
}

\author{
PALLAB GAYEN \\ Department of Chemistry, Raja Peary Mohan College, West Bengal, India. \\ *Corresponding author E-mail: pallabinorganic@gmail.com
}

http://dx.doi.org/10.13005/ojc/370530

(Received: June 09, 2021; Accepted: September 09, 2021)

\section{ABSTRACT}

1-Alkyl-2-(arylazo)imidazole(RaaiR') exists in trans-structure about $-\mathrm{N}=\mathrm{N}$ - bond at ambient condition. Upon optical excitation in UV region the trans-RaaiR' isomerises to cis-RaaiR'. The photochromism is very susceptible to internal substituents and external environment like solvent polarity, viscosity and presence of innocent foreign molecule. The changes from cis-to-trans occurs slowly in visible light excitation it has significantly faster rate at higher temperature. In this work we have studied the effect of silver nanoparticle on the photochromic activity of RaaiR'. The quantum yield of the photoisomerisation is dropped by 9-27\% in inclusion phase AgNPs@RaaiR' than free state and the order of rate is: free state > silver nano particle. The activation energy $\left(E_{a}\right)$ of cis to trans isomerisation is also diminished compared to free state of photochrome.

Keywords: Photochromism, Arylazoimidazole, Nanoparticle, Activation energy.

\section{INTRODUCTION}

Due to exceptional values and possible potential applications in technological aspects, the method of production and identification of nanostructured substances earning a lot of importance. The structure of these nano influences on its optical, electronic, magnetic, and catalytic properties. So, the most important target in nano particle preparation is to monitor the structure and morphology of the particle..$^{1-4}$

The term photochromism is applied to explain the mechanism of colour change caused by photon energy, and a reversible changes in colour is observed in all photochromic substances by the thermal process or by particular quantum energy. Following the above mentioned possible applications, photochromic substances are being attempted to be applied in sensors and optical shutters. ${ }^{5-8}$

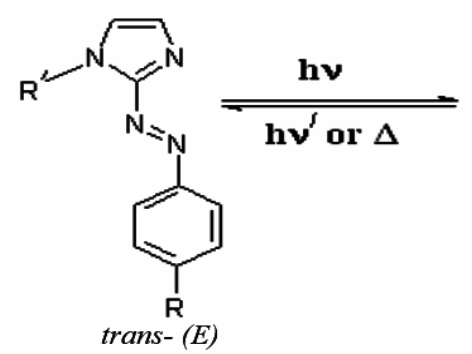<smiles>[R]c1ccc(/N=N\c2nccn2[R])cc1</smiles>

Scheme. 1. Isomeric change of RaaiR

This is an Open Access article licensed under a Creative Commons license: Attribution 4.0 International (CC- BY). Published by Oriental Scientific Publishing Company @ 2018 
We have been exploring the trans-cis photoisomerisation of RaaiR' (Scheme 1) and the compounds with different metal ${ }^{9-16}$. The impact of microenvironment (in presence of micelles, reverse micelle, aromatic acids, phenols, hydrocarbons) on the photoisomerisation of Raai $R^{\prime}$ has been examined for last few years ${ }^{17-21}$. Use of light energy as stimulant has huge dominance over other influences like thermal, magnetic, mechanical, electrical, redox etc. Photochromism is a reversible alteration process between two isomeric structures having different absorption spectral curve ${ }^{22}$. The rate of photo-transformation and quantum yields of trans-cis isomeric changes of RaaiR' are affected by inner environment such as-nature of substituents, binding to metals, steric and stereoelectronic effect, hydronation etc and the extrinsic environment such as solvent (concentration, dipolar nature, weak forces and interaction due to oppositely charged ion), On considering the importance of MNPs \& the application of photochromism we are now interested to study the efficacy of photochromism of Raai $R^{\prime}$ when it is the surface of silver nano particles.

\section{EXPERIMENTAL}

\section{Materials and Methods}

The photochromic molecules were made by reported method ${ }^{23}$ (Scheme 2). SRL, India had supplied Silver nitrate, sodium borohydride, Polyvinylpyrrolidone (PVP) and other reagents. We have purified 2-propanol by distillation (SRL, India) before use.

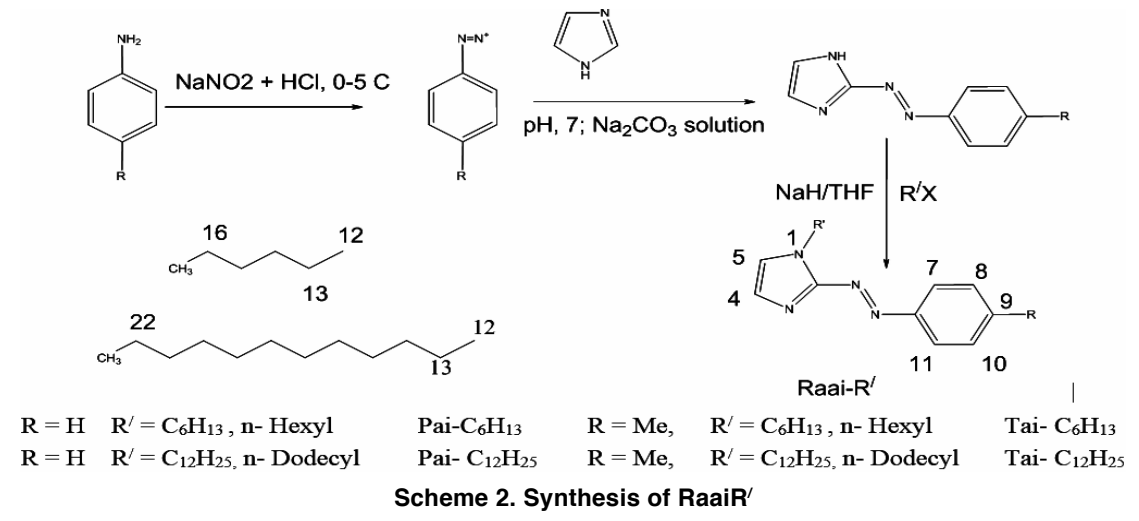

\section{Synthesis of silver nanoparticles covered with} 1-alkyl-2-(arylazo)imidazoles

Silver nanoparticles (AgNPs) covered with the photochromic molecules (RaaiR') were made by following method which has been already reported ${ }^{24} .10 \mathrm{~mL} 1.0 \mathrm{mM}$ of silver nitrate $\left(\mathrm{AgNO}_{3}\right)$ in 2-propanol was mixed to $10 \mathrm{~mL} 5.0 \mathrm{mM}$ of sodium borohydride $\left(\mathrm{NaBH}_{4}\right)$, under stirring condition in presence of Polyvinylpyrrolidone (PVP) which has been used as stabilizing agent. The preparation was done in dark and ice cold condition was maintained. The growth of Ag NPs was established by absorption spectra of the yellow solution obtained. Propanol solution of RaaiR' (1.0 mM) was poured to the reaction mixture and stirring was continued for some times. Few drops of $\mathrm{NaBH}_{4}$ were added in drops at ice cold condition. The solution was allowed for equilibrium at normal room temperature for $\sim 20$ hours. Final mixture was added to alcohol to eliminate PVP and the unwanted chemicals, then filtered. The solid mass was obtained and dried. The residue was mixed to $25 \mathrm{~mL}$ of diethyl ether. The excess Raai $R^{\prime}$ is soluble in $\left(\mathrm{C}_{2} \mathrm{H}_{5}\right)_{2} \mathrm{O}$. Then hazy $\left(\mathrm{C}_{2} \mathrm{H}_{5}\right)_{2} \mathrm{O}$ was centrifuged and the precipitate was recollected. Ag-(RaaiR)-NP-PVP was obtained as a deep brown solid. The size and shape of the particle was confirmed from the SEM pictures.

\section{Photometric estimations}

Spectrophotometer (Perkin Elmer Lambda 25 UV/Vis) and spectrofluorimeter (Perkin Elmer LS55) was used. Quantum yields ( $\phi$ ) are calculated by the relation,

$$
v=\left(\phi I_{0} N\right)\left(1-10^{-A b s o r b a n c e}\right)
$$

Where $v=$ rate of photo isomerisation, $\mathrm{I}_{0}=$ photon flux, $\mathrm{V}=$ volume, Abs = initial absorbance at the azobenzene (quantum yield is 0.11 of $\pi-\pi^{*}$ transition $)^{25}$. 


\section{RESULTS AND DISCUSSIONS}

\section{Photoisomerisation from trans to cis conversion}

The spectra of RaaiR' in 2-propanol solution gives an absorption band $\sim 360-390$ nanometer(molar absorptivity of the order $10^{4} / \mathrm{Mole} /$ $\mathrm{cm}$ ) and a tail extending to 452 nanometer. The high energy intense band is for $\pi-\pi^{*}$ transitions, while tail is for $n-\pi^{*} \operatorname{transition}^{13}$. Excitation of ultraviolet ray to the propanol solution of the compound changes the absorption spectrum as shown in Fig. 1. It is noticed that by excitation with ultra violet ray trans-cis photoisomerisation has proceeded and concentration of cis structure is arrived to an expected percentage.

The UV light is passed to the mixture of RaaiR' and AgNPs. The photoisomerisation rate changes data are noted in Table 1 and corresponding figure are given in Fig. 2. In presence of AgNPs, the rate of isomerisation is reduced by $10-33 \%$ than that of free molecular data. This reaction rate decreases compared to 2-propanol solution. The effect of variation of AgNPs on isomerisation rate have been studied (Table 1) and Ag nanoparticle have a significant effect on quantum yield. The photoresistance of RaaiR' even in presence of AgNPs are examined by repetition of trans-cis cycle.

Azobezene-thiol-modified gold nanoparticles exhibit size-reduction induced ferromagnetism and significant photo-magnetic effects ${ }^{26}$. The isomeric changes of azobenzene derivatives by light confined in AuNP aggregates leading to a redshift of the surface plasmon band in the ultra violet-visible spectrum $^{27}$. AgNPs in presence of added Raai ${ }^{\prime}$ has been examined by red shift of surface plasmon band (Fig. 3). The SEM of silver nanoparticle (Fig. 4) and SEM of silver nanoparticle in presence of RaaiR' (Fig. 5) support the aggregation of NPs. The rates of photoisomerisation and quantum yield were decreasing in presence of AgNPs. This has implicated that photochrome, RaaiR', has been associated with AgNPs and the increase in effective photochromic mass and rotor volume may be responsible for effective decrement of photoisomerisation rates and quantum yields.

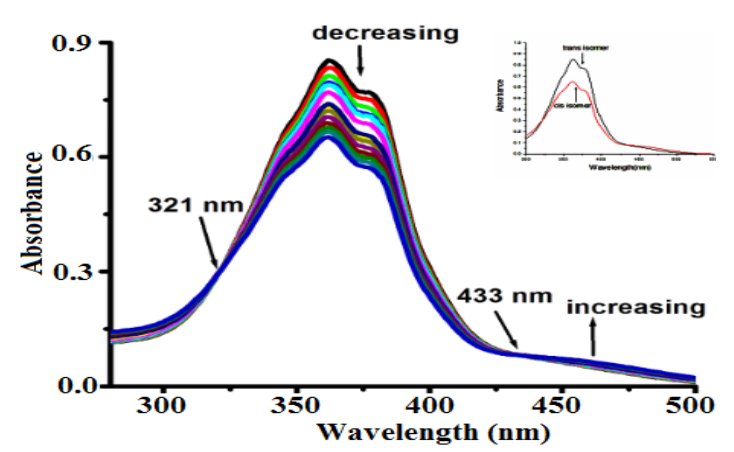

Fig. 1. Spectral changes of Pai- $\mathrm{C}_{6} \mathrm{H}_{13}$ in 2-Propanol upon continual excitation at a wavelength of $364 \mathrm{~nm}$ at $3 \mathrm{~min}$

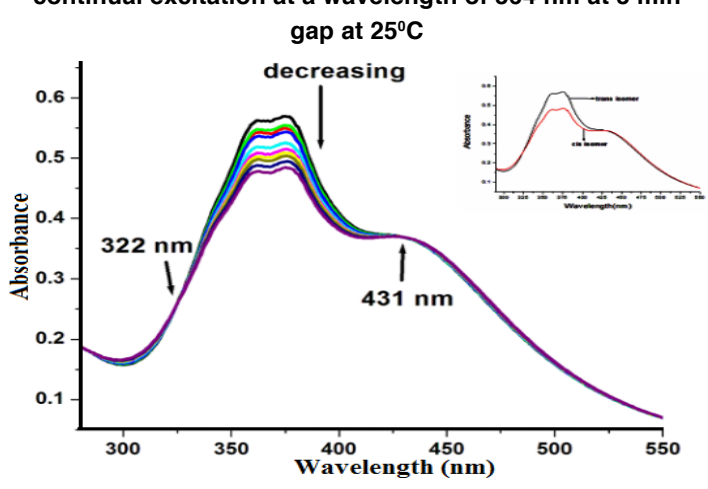

Fig. 2. Spectral changes of Pai- $\mathrm{C}_{12} \mathrm{H}_{25}$ in 2-Propanol on the surface of Ag-nano upon continual excitation at a wavelength of $366 \mathrm{~nm}$ at $3 \mathrm{~min}$ gap at $25^{\circ} \mathrm{C}$

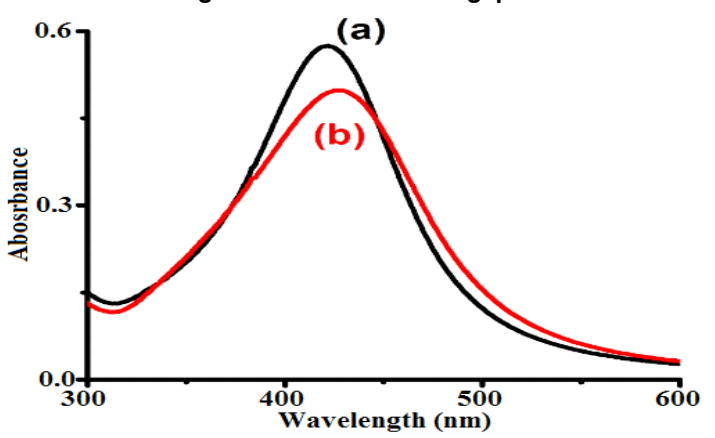

Fig. 3. (a) Ultra Violet spectra of Ag nano particles $(\lambda \max =421 \mathrm{~nm})(\mathrm{b})$ UV spectra of AgNPs in presence of Pai- $\mathrm{C}_{12} \mathrm{H}_{25}(\lambda \max =427 \mathrm{~nm})$

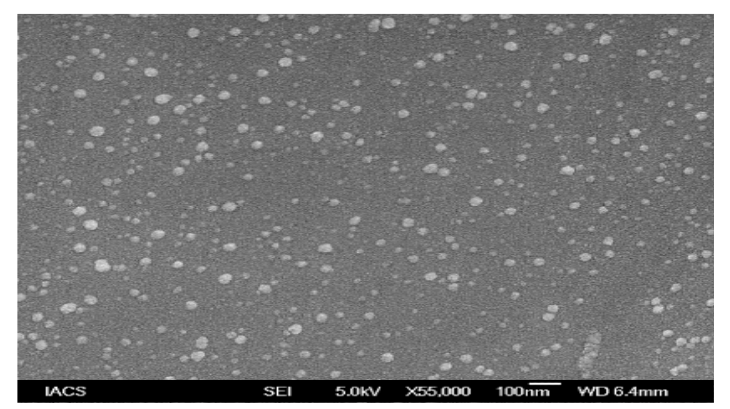

Fig. 4. SEM images of Ag nanoparticles (nano range:20-30nm) 


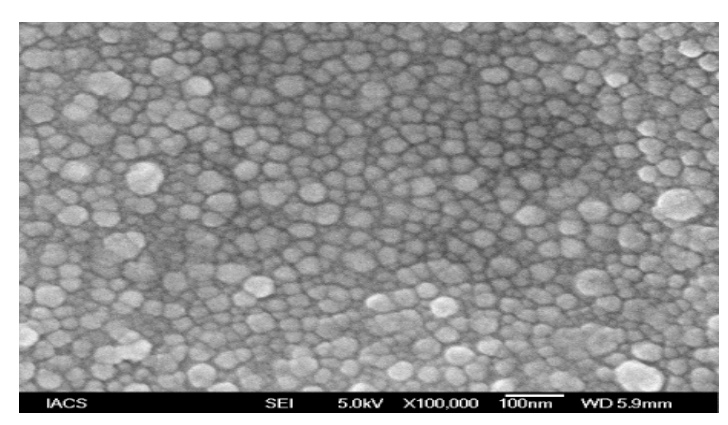

Fig. 5. SEM image of AgNPs in presence of Pai- $\mathrm{C}_{12} \mathrm{H}_{25}$ Thermal isomerisation, cis $\rightarrow$ trans

RaaiR' has a steady trans structure at ambient atmosphere. By ultra violet ray RaaiR' have been changes to cis one. Cis structure is changes to trans one by reverse process by visible light $(450 \mathrm{~nm})$. We get relatively faster rate when the thermal change is done in dark. We can calculate the rate constant $(\mathrm{k})$ of thermal reverse process by the relation $A b s(t)=$ $\left(\mathrm{Abs}_{\mathrm{t}}-\mathrm{Abs}_{\mathrm{E}}\right) \mathrm{e}^{-\mathrm{kt}}+\mathrm{Abs}_{\mathrm{c}}$, here $\mathrm{Abs}_{\mathrm{c}}=$ absorbance of cis form and $\mathrm{Abs}_{\mathrm{t}}=$ absorbance of trans form.

Cis product is changes thermally to trans product in the dark at a temperature ranges of (298313) $\mathrm{K}$. We get the energy of activation $\left(E_{a}\right)$ and the pre-exponential factor $(A)$ using relation $k=A \cdot e^{-E a /}$ $\mathrm{RT}$, here $\mathrm{k}=$ reaction rate, $\mathrm{R}=$ ideal gas constant, and $T=$ temp. We get the values of the activation free energy $\left(\Delta G^{*}\right)$ and the activation entropy $\left(\Delta S^{*}\right)$ by using the equations.

$$
\Delta \mathrm{G}^{*}=\mathrm{E}_{\mathrm{a}}-\mathrm{RT}-\mathrm{T} \Delta \mathrm{S}^{*} \text { and } \Delta \mathrm{S}^{\star}=\left[\ln \mathrm{A}-1-\ln \left(\mathrm{k}_{\mathrm{B}} \mathrm{T} / \mathrm{h}\right) / \mathrm{R}\right.
$$

Here $\mathrm{k}_{\mathrm{B}}=$ Boltzmann's constant, $\mathrm{h}=$ Plank's constant

Table 1: Photoisomerisation of $\operatorname{RaaiR}^{\prime}\left(R^{\prime}=-C_{n} H_{2 n+1}\right.$ where $\left.n=6,12\right)$ in presence Ag-nano

\begin{tabular}{lcccc}
\hline Compound & $\lambda \pi, \pi^{*}$ (nanometer) & $\begin{array}{c}\text { Isosbestic Point } \\
\text { (nanometer) }\end{array}$ & $\begin{array}{c}\text { Rate of trans } \rightarrow \text { cis } \times 10^{8} \\
\text { (second }{ }^{-1} \text { ) }\end{array}$ & $\begin{array}{c}\text { Quantum Yield } \\
\text { (trans } \rightarrow \text { cis) }\end{array}$ \\
\hline Pai- ${ }_{6} \mathrm{H}_{13}$ & 364 & 327,445 & 2.74 & 0.135 \\
${\text { Tai- } \mathrm{C}_{6} \mathrm{H}_{13}}_{\text {Pai- } \mathrm{C}_{6} \mathrm{H}_{13} @ \text { Ag-nano }}^{365}$ & 366 & 336,433 & 2.69 & 0.106 \\
${\text { Tai- } \mathrm{C}_{6} \mathrm{H}_{13} @ \text { Ag-nano }}_{\text {Pai- }{ }_{12} \mathrm{H}_{25}}^{365}$ & 366 & 334,437 & 2.09 & 0.099 \\
Tai- $\mathrm{C}_{12} \mathrm{H}_{25}$ & 366 & 326,447 & 1.82 & 0.085 \\
Pai- $\mathrm{C}_{12} \mathrm{H}_{25} @$ Ag-nano & 366 & 336,433 & 2.17 & 0.102 \\
Tai- $\mathrm{C}_{12} \mathrm{H}_{25} @$ Ag-nano & 367 & 332,441 & 1.93 & 0.095 \\
\hline
\end{tabular}

The energy of activation for thermal isomeric changes was measured by plotting $\mathrm{k}$ values (Table 2, Fig. 6). Cis-RaaiR' is more polar than trans-structure. NPs are electronically more negative than atom and can be associated with electronically deficient part in the molecule and are capable to execute electrostatic interaction ${ }^{28}$. We

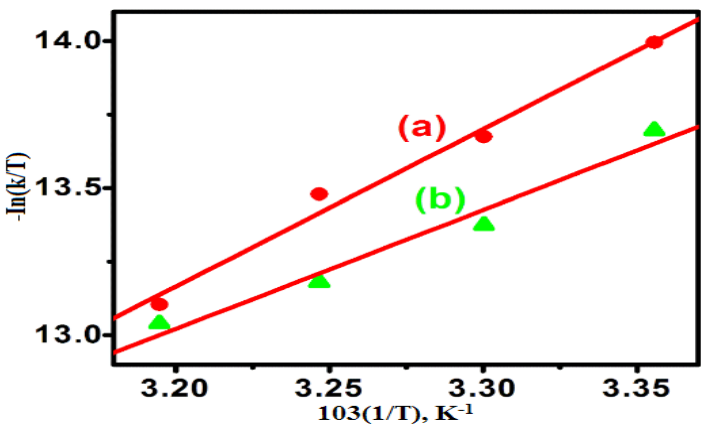

Fig. 6. Eyring plots for cis $\rightarrow$ trans conversion of (a) Pai- $\mathrm{C}_{6} \mathrm{H}_{13}$, (b) Pai- $\mathrm{C}_{6} \mathrm{H}_{13} @ f$ Ag nanoparticles assume that azoimidazolyl part of photochrome being an electron deficient part in the molecule may associate about AgNPs and is leading to increment of effective mass and volume of photochrome. A cartoon is shown in Scheme 3 which describes the plausible pathway of photochromic changes of RaaiR' on the surface of nanoparticle.
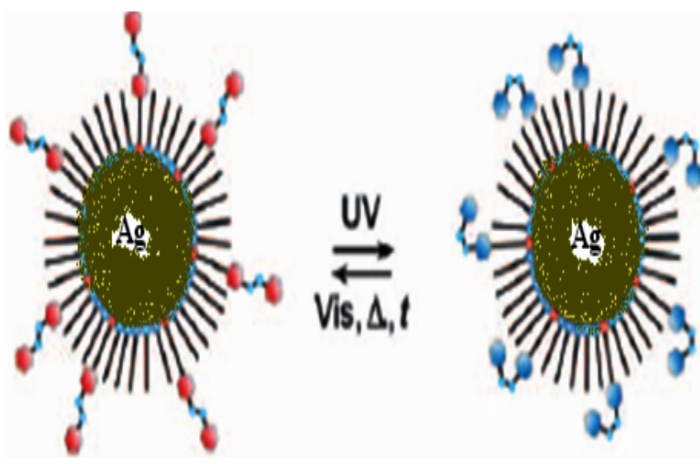

Scheme 3 
Table 2: Thermal data for cis $\rightarrow$ trans

\begin{tabular}{|c|c|c|c|c|c|c|}
\hline Product & Temp(K) & Rate $\mathrm{c} \rightarrow$ t process $\times 10^{4}\left(\mathrm{sec}^{-1}\right)$ & $\begin{array}{c}\mathrm{E}_{\mathrm{a}}, \\
\mathrm{kJ} / \text { mole }\end{array}$ & $\begin{array}{c}\Delta \mathrm{H}^{*} \\
\mathrm{~kJ} / \mathrm{mole}\end{array}$ & $\begin{array}{c}\Delta \mathrm{S}^{*} \\
\mathrm{~J} / \mathrm{mole} / \mathrm{K}\end{array}$ & $\begin{array}{c}\Delta \mathrm{G}^{*} \\
\mathrm{~kJ} / \mathrm{mole}\end{array}$ \\
\hline \multirow[t]{4}{*}{ Pai- $\mathrm{C}_{6} \mathrm{H}_{13}$} & 298 & 2.2 & 43.73 & 41.19 & -176.26 & 93.68 \\
\hline & 303 & 3.26 & & & & \\
\hline & 308 & 4.27 & & & & \\
\hline & 313 & 5.22 & & & & \\
\hline \multirow[t]{4}{*}{ Tai- $\mathrm{C}_{6} \mathrm{H}_{13}$} & 298 & 3.5 & 42.21 & 39.67 & -177.35 & 92.49 \\
\hline & 303 & 5.77 & & & & \\
\hline & 308 & 6.7 & & & & \\
\hline & 313 & 8.21 & & & & \\
\hline \multirow[t]{4}{*}{ Pai- $\mathrm{C}_{6} \mathrm{H}_{13} @ \mathrm{Ag}$-nano } & 298 & 2.41 & 41.26 & 38.73 & -184.19 & 93.59 \\
\hline & 303 & 3.24 & & & & \\
\hline & 308 & 4.25 & & & & \\
\hline & 313 & 5.3 & & & & \\
\hline \multirow[t]{4}{*}{ Tai- $\mathrm{C}_{6} \mathrm{H}_{13} @$ Ag-nano } & 298 & 4.55 & 30.37 & 27.81 & -215.49 & 92.04 \\
\hline & 303 & 5.81 & & & & \\
\hline & 308 & 6.76 & & & & \\
\hline & 313 & 8.23 & & & & \\
\hline \multirow[t]{4}{*}{ Pai- $\mathrm{C}_{12} \mathrm{H}_{25}$} & 298 & 2.47 & 42.01 & 39.46 & -181.89 & 93.66 \\
\hline & 303 & 3.11 & & & & \\
\hline & 308 & 4.12 & & & & \\
\hline & 313 & 5.39 & & & & \\
\hline \multirow[t]{4}{*}{ Tai- $\mathrm{C}_{12} \mathrm{H}_{25}$} & 298 & 3.8 & 38.06 & 35.5 & -191.48 & 92.55 \\
\hline & 303 & 4.46 & & & & \\
\hline & 308 & 6.88 & & & & \\
\hline & 313 & 7.53 & & & & \\
\hline \multirow[t]{4}{*}{ Pai- $\mathrm{C}_{12} \mathrm{H}_{25} @$ Ag-nano } & 298 & 2.63 & 36.81 & 34.25 & -198.46 & 93.38 \\
\hline & 303 & 3.47 & & & & \\
\hline & 308 & 4.32 & & & & \\
\hline & 313 & 5.37 & & & & \\
\hline \multirow[t]{4}{*}{ Tai- $\mathrm{C}_{12} \mathrm{H}_{25} @ \mathrm{Ag}$-nano } & 298 & 1.2 & 28.27 & 25.75 & -233.55 & 95.35 \\
\hline & 303 & 1.42 & & & & \\
\hline & 308 & 1.88 & & & & \\
\hline & 313 & 2.01 & & & & \\
\hline
\end{tabular}

\section{CONCLUSION}

The trans-cis changes of Raai $R^{\prime}$ on light excitation are examined along with the AgNPs. Backward process i.e the changes of cis geometry to trans geometry is relaxed by light. By applying heat the process can be permitted. Trans-to-cis photo isomerisation rate is reduced on the nano particle surface. Increase the nano particle concentration decreases the rate. It is due to the electrostatic attraction between ligand and nanoparticle.

\section{ACKNOWLEDGEMENT}

I am very much thankful to my Sir, my research guide Professor Chittaranjan Sinha for giving me all types of facility (instrumental and laboratory) to complete the work. Without his guidance and constant support it was quite impossible to do the work. I am also thankful to one of my best student Miss Sunanda Dey for helping me during this work.

\section{Conflict of interest}

Author of this paper have no conflict of interest.

\section{REFERENCES}

1. Link, S.; Mohamed, M.B.; El-Sayed, M.A. J. 4. Ahmadi, S.; Wang, Z.L.; Green, T.C.; Phys. Chem. B., 1999, 103, 3073.

2. Ichikawa, M. J. Am. Chem. Soc., 2001, 123, 3373.

3. Gates, B.; Yin, Y.; Xia, Y. J. Am. Chem. Soc., 2000, 122, 582.

Henglein, A.; El-Sayed, M.A. Science., 1996, 272, 924.

5. Kobatake, S.; Yamada, T.; Uchida, K.; Kato, N.; Irie, M. J. Am. Chem. Soc., 1999, 121, 2380. 
6. Fraysse, S.; Coudret, C.; Launay, J. P. Eur. J. Inorg. Chem., 2000, 7, 1581.

7. Kondo, Y.; Inouye, H.; Fujiwara, S.; Suzuki, T.; Mitsuyu, T.; Yoko, T.; Hirano, K. J. Appl. Phys., 2000, 88, 1244.

8. Tulyakova, E. V.; Vermeersch, G.; Gulakova, E. N.; Fedorova, O. A.; Fedorov, Y. V; Micheau, J. C; Delbaere, S. Chem. Eur. J., 2011, 17.

9. Otsuki, J.; Suwa, K.; Narutaki, K.; Sinha, C.; Yoshikawa, I.; Arakil, K. J. Phys. Chem. A., 2005, 109, 8064.

10. Otsuki, J.; Suwa, K.; Sarker, KK.; Sinha, C; J. Phys. Chem. A., 2007, 111, 1403.

11. Sarker, KK.; Halder Saha, S.; Banerjee, D.; Mondal, TK.; Paital, AR.; Nanda, PK.; Raghavaiah, P.; Sinha, C.; Inorg. Chim. Acta., 2010, 363, 2955.

12. Sarker, KK.; Sardar, D.; Suwa, K.; Otsuki, J.; Sinha, C. Inorg. Chem., 2007, 46, 8291.

13. Sarker, KK.; Chand, BG.; Suwa, K.; Cheng, J.; Lu, HT.; Otsuki, J.; Sinha, C. Inorg. Chem., 2007, 46, 670.

14. Pratihar, P.; Mondal, TK.; Patra, AK.; Sinha, C. Inorg. Chem., 2009, 48, 2760.

15. Saha, G.; Datta, P.; Sarkar, KK.; Saha, R.; Mostafa, G.; Sinha, C. Polyhedron., 2011, 30, 614.

16. Mallick, D.; Nandi, A.; Datta, S.; Sarker, KK.; Mondal, TK.; Sinha, C. Polyhedron., 2012, 31, 506.
17. Gayen, P.; Sarker KK.; Sinha C. Colloids and Surfaces A., 2013, 429, 60-66.

18. Gayen, P.; Sinha, C. J. Indian Chem. Soc., 2013, 90, 751-762.

19. Gayen, P.; Sinha, C. Spectrochimica ActaPart A., 2013, 104, 477-485.

20. Gayen P.; Sinha C. Spectrochimica Acta- Part A., 2012, 98, 116-121.

21. Gayen, P.; Sinha C. J. Lumin., 2012, 132, 2371-2377.

22. Dürr,H; Bounas-Laurent (Eds.),H. Photochromism: Molecules and Systems. Elsevier: Amsterdam., 2003.

23. Mallick, D.; Nandi, A.; Datta, S.; Sarker, K.K.; Mondal, T.K.; Sinha, C. Polyhedron., 2012, 31, 506-514.

24. Nishi H; Kobatake, S. Macromolecules., 2008, 41, 3995.

25. Zimmerman, G.; Chow, L.; Paik, U. J. Am. Chem. Soc., 1958, 80, 3528.

26. Suda, M.; Kameyama, N.; Ikegami, A.; Suzuki, M.; Kawamura, N.; Einaga, Y. Polyhedron., 2009, 28, 1868-1874.

27. Yoon, JH.; Yoon, S. Phys. Chem. Chem. Phys., 2011, 13, 12900-12905.

28. Moores, A.; Goettmann, F. New J. Chem., 2006, 30, 1121. 